\title{
La integración del alumno al mundo laboral. El paradigma de la Inteligencia Emocional en el marco del Espacio Europeo de Educación Superior (EEES)
}

\author{
Carlos Del Val Merino \\ Dirección de Comunicación y Patrocinio del Instituto Cervantes \\ cval_exmba2002@yahoo.es
}

Recibido: 08/10/2012

Aceptado: 23/01/2013

\begin{abstract}
Resumen
El cada vez más dinámico entorno empresarial caracterizado por el efecto de la globalización, ha potenciado el desarrollo de nuevos estilos de gerencia que demanda nuevos perfiles de universitarios necesarios para cubrir las exigencias del mercado.

Este brusco cambio exige un obligado acercamiento entre las demandas reales de las empresas y el nuevo marco del Espacio Europeo de Educación Superior (EEES), donde el alumno se debe convertir en el protagonista principal, ya que se enfrentará a situaciones laborales totalmente desconocidas.

La Inteligencia Emocional se convierte en una disciplina indispensable a adoptar por las Instituciones Universitarias en sus planes de estudio para dotar a los alumnos de una educación integral donde se conjuguen las competencias académicas con inteligencias no estrictamente cognitivas que les ayuden a desarrollarse a nivel personal y profesional.

Palabras clave: Espacio Europeo de Educación Superior (EEES), Universidad, Inteligencia Emocional, mercado

\section{Students' Integration into the Workplace. The Paradigm of Emotional Intelligence in the Context of the European Higher Education Area (EHEA)}

\begin{abstract}
The dynamic business environment characterized by the globalization effect has developed new Business management styles that demand new university profiles needed to cover the market demands.

This radical change requires a mandatory approach between the real business demands and the new European Higher Education Area (EHEA) framework, where student must become the main protagonists, due to the fact that they will face working situations completely unknown by them.

Emotional Intelligence becomes an essential discipline to be adopted by higher education institutions in their curricula to provide students with a comprehensive education which combines academic skills with strictly no cognitive intelligences that will help them to grow personally and professionally.

Keywords: European Higher Education Area (EHEA), University, Emotional Intelligence, Market

\section{Referencia normalizada}

DEL VAL MERINO, Carlos (2013): "La integración del alumno al mundo laboral. El paradigma de la Inteligencia Emocional en el marco del Espacio Europeo de Educación Superior (EEES)". Estudios sobre el Mensaje Periodístico. Vol. 19, Núm. especial marzo, págs.: 129-138. Madrid, Servicio de Publicaciones de la Universidad Complutense.
\end{abstract}

Sumario: 1. Introducción. 2. El modelo de competencias en el EEES y la IE; 2.1. La Inteligencia Emocional (IE) como factor determinante en el modelo de competencias genéricas; 2.2. La IE y las competencias socio-emocionales de los estudiantes; 2.3. La inteligencia emocional en el contexto empresarial. 3. Metodología y desarrollo. 4. Conclusiones. 5. Referencias bibliográficas. 


\section{Introducción}

Los modelos educativos actuales de gran número de universidades españolas, inmersos en una vorágine de transformaciones impulsadas por el proceso de Bolonia, quedan muy lejos de ser sistemas eficientes que transformen a sus estudiantes en trabajadores empleables que puedan competir, no sólo en los tradicionales mercados occidentales, sino sobre todo en los nuevos mercados emergentes que empiezan a despertar en un entorno de globalización y competitividad cada vez más cambiante.

Cada vez son más los casos en los que no sólo los propios empresarios, sino sobre todo los estudiantes, se quejan del distanciamiento cada vez mayor existente entre la formación universitaria y las necesidades reales de las empresas y del exigente mercado laboral.

Con el fin de paliar estos problemas y con el objeto de potenciar la Europa del conocimiento que armonice los sistemas de educación superior, en mayo de 1998 se realiza la Declaración de la Sorbona como primer paso para la consolidación y desarrollo de las dimensiones intelectuales, culturales, sociales y técnicas de Europa, teniendo las universidades un papel decisivo y relevante en el desarrollo de estos aspectos.

A partir de entonces se toma la iniciativa de crear un Espacio Europeo de Educación Superior (EEES) cuyos grandes retos planteados desde su inicio eran: "el aumento de la demanda de formación superior, la internacionalización de la educación y la investigación, la cooperación entre las universidades y empresas, la multiplicación de los lugares de producción de conocimientos, la reorganización de los conocimientos, y por último la aparición de nuevas expectativas" (Comisión Europea, 2003, web) de modo que se favorezca la movilidad y las oportunidades de empleo, y en el que identidades nacionales e intereses comunes puedan relacionarse y reforzarse fomentando el desarrollo global de Europa.

Un año después, la Declaración de Bolonia adherida a los principios establecidos en la Declaración de la Sorbona, sentó las bases para la construcción del EEES, fijándose entre otros objetivos: la flexibilidad en las titulaciones; la movilidad y libre circulación de estudiantes y profesores; la cooperación europea para asegurar un sistema de calidad...

La Declaración de Bolonia reconocía que la consecución de estos objetivos requería de esfuerzos permanentes de planificación, produciéndose el paso del aprendizaje por objetivos del profesor al aprendizaje de competencias por el estudiante" (Pedró, F., ,2005: 19). Estos grandes cambios no se improvisan, precisándose "un periodo de acomodación, de superación de inercias ancestrales, de individualismo, de formación docente para el trabajo en equipo y con estrategias creativas y de concienciación del alumnado". (Torre, De la., 2009: web) y un seguimiento que asegurara su implantación efectiva, incluyendo la formación en competencias y destrezas para que se produjera un acercamiento real entre universidad y empresa.

Estos nuevos conocimientos y destrezas a adquirir por parte de los universitarios no se deben circunscribir al ámbito de conocimientos meramente académicos y cognitivos, ya que hasta el momento éstos no han conseguido una completa adaptabilidad a la empresa, siendo necesario un complemento indispensable que ayude a los alumnos a adquirir nuevas habilidades y competencias que les hagan ser más empleables. 
Como objetivos del presente artículo cabría destacar la concienciación sobre la importancia que supone el acercamiento entre las instituciones universitarias públicas españolas y el tejido empresarial, evidenciando la importancia que tiene el hecho de la rápida adaptabilidad de los estudios universitarios dentro del marco del EEES, a las demandas y necesidades reales de las empresas.

Por otra parte se quiere mostrar la existencia de otra tipología de competencias, aparte de las ya integradas en algunos planes de estudio, y que tienen más que ver con el desarrollo de competencias y aptitudes emocionales, y no tanto con la adquisición de conocimientos académicos.

Como objetivos específicos se busca conocer la percepción sobre el estado del EEES y los avances producidos en su ámbito de acción, tanto de estudiantes que actualmente cursan los últimos cursos de grado, como de profesores que imparten docencia en grado en universidades públicas españolas, ya que ellos se convierten en los principales actores y precursores de esta transformación académica.

También se desea conocer la opinión que tienen estos colectivos sobre los potenciales beneficios que supondría la incorporación de materias relacionadas con la inteligencia emocional complementando los actuales planes de estudio, y las ventajas que ésto supondría para los estudiantes.

\section{EI modelo de competencias en el EEES y la IE}

El concepto de competencia laboral emergió con fuerza en los años 80 en países desarrollados donde existía una barrera y distanciamiento entre las cualificaciones ofrecidas por los sistemas educativos y las auténticas necesidades del entorno laboral.

A fecha de hoy se han desarrollado varios estudios sobre el modelo de docencia que facilite el desarrollo de competencias propuesto en el proceso de Bolonia, y que pretende ayudar a los estudiantes a adaptarse de forma más eficiente al entorno profesional.

Las competencias las podríamos entender como aquellos conocimientos y habilidades, actitudes y valores que adquieren los alumnos y que les capacitan para la resolución de problemas no sólo en el contexto académico, sino también profesional o personal. Cada competencia se construye a través de la combinación de habilidades cognitivas y prácticas, conocimiento (incluyendo el conocimiento táctico), motivación, valores, actitudes, emociones y otros componentes sociales y conductuales.

En concordancia con las nuevas premisas marcadas por la reforma del EEES, existen varias clasificaciones en cuanto a competencias se refiere, aunque la más usada es la que las divide en competencias transversales o genéricas, que son compartidas por todas las materias o ámbitos de conocimiento (instrumentales, interpersonales, sistémicas), y competencias específicas (académicas, disciplinares, profesionales) que "se refieren a aquellas relacionadas con un área de conocimiento" (Gonzalez Ferreras, 2006: 417-418).

En línea con Xavier Garagorri (2007), la introducción en el diseño curricular y en los procesos de aprendizaje de estas nuevas competencias, se convierte en un desafío y una oportunidad para repensar el sentido de la educación, dejando el objetivo de ser la acumulación exclusiva de conocimientos académicos y pasando a ser una combina- 
ción de éstos con el desarrollo de una serie de competencias relevantes que se necesitan para enfrentarse a los problemas y para poder integrarse en el mercado laboral.

Se hace necesario un aprendizaje creativo que "hace referencia al conocimiento construido con la implicación activa del sujeto, desde su planificación hasta su internacionalización, caracterizado por la motivación intrínseca" (Torre, De la., S. 1993: 272). Un modelo orientado a la acción cuya finalidad "es la adquisición de habilidades" (Sangra, A. et al., 2008: 50) siendo el elemento central de la estructura las actividades, ya que actúan como hilo conductor.

\subsection{La Inteligencia Emocional (IE) como factor determinante en el modelo de competencias genéricas}

La inteligencia emocional es un término muy controvertido sobre el que se ha escrito mucho, especialmente desde mediados del siglo pasado hasta la fecha, siendo varias las definiciones que intentan determinar este concepto de importancia creciente en diversos campos de índole social y económica.

Varios autores y psicólogos llevan investigando y definiendo el término de inteligencia emocional desde hace varias décadas. De todos es conocido el Best Seller de Daniel Goleman (2007) quien habla de la importancia de los factores emocionales y sociales en los individuos, afirmando que existen habilidades más importantes que la inteligencia académica a la hora de alcanzar un mayor bienestar laboral, personal, académico y social.

Otros estudiosos definen la inteligencia emocional de un modo más claro y conciso como: "la capacidad para supervisar los sentimientos y las emociones de uno/a mismo/a y de los demás, de discriminar entre ellos y de usar esta información para la orientación de la acción y el pensamiento propios" (Salovey y Mayer, 1990: 189).

No obstante, variables como la inteligencia emocional y empresa han sido conceptos que durante muchos años se han movido en planos de la realidad distintos. Las emociones, que se han movido en el plano de los sentimientos, eran algo que antaño se ocultaban por considerarse en muchos casos signo de debilidad del trabajador, y la empresa se ha concebido tradicionalmente como un ente objetivo, siendo una institución ligada al beneficio y por tanto que requiere de criterios económicos y no emocionales para su buen gobierno.

Los estudios realizados en el ámbito de la psicología se han centrado en la relación de la inteligencia emocional con variables como el estrés, la ansiedad y la depresión, siendo las siguientes las principales conclusiones a las que han llegado estos estudios:

- Las personas con elevada inteligencia emocional presentan mayor satisfacción con la vida, mayor calidad y cantidad de relaciones sociales, más éxito académico y mayor conducta prosocial.

- Reparar los estados emocionales negativos, prolongar los positivos, y experimentar con claridad las emociones, es un potente predictor de ajuste psicológico.

- Una elevada inteligencia emocional proporciona más competencias para afrontar las situaciones estresantes de forma adaptativa. 
Basándonos en estas conclusiones se podría adelantar que existen grandes ventajas si se aplicaran políticas académicas dentro del contexto universitario que ayuden a la implementación de materias relacionadas con la formación en capacidades emocionales, y que paulatinamente e independientemente del tipo de estudios impartidos, vayan complementando no sólo a los planes curriculares de cada especialidad, sino también a los planes de carrera profesional de las propias empresas.

\subsection{La IE y las competencias socio-emocionales de los estudiantes}

El coeficiente intelectual o CI (mide las habilidades cognitivas de una persona), está pasando a ocupar un segundo plano por debajo de la inteligencia emocional.

"La correlación existente entre el CI y el nivel de eficacia que muestran las personas en el desempeño de su profesión no supera el $25 \%$, aunque un análisis más detallado revela que esta correlación no suele superar el $10 \%$ y a veces es incluso inferior al 4\%" (Goleman, 2000: 39).

Hay veces en las que nos preguntamos el motivo por el que estudiantes con un brillante expediente académico y elevado CI fracasan en sus tareas profesionales e incluso en las personales, y por qué existen otros estudiantes que con resultados académicos menos brillantes triunfan en muchas parcelas de la vida, especialmente en la profesional.

Por supuesto que la pericia (entendida como el conjunto de conocimientos especializados y de habilidades prácticas) es la competencia específica que capacita al estudiante para desempeñar adecuadamente un determinado trabajo (especialmente una labor técnica), pero es evidente que no es ni lo único ni lo más importante para alcanzar el éxito profesional y personal.

La investigación realizada a nivel mundial por el Consortium for Research on Emotional Intelligence in Organizations (1999: web) arrojó un resultado sorprendente vinculado al cociente de éxito. El éxito profesional se debe en un $23 \%$ a las capacidades intelectuales y en un $77 \%$ a nuestras aptitudes emocionales.

Entre el $40 \%$ y el $50 \%$ de los universitarios dejan la carrera, contándose entre las causas principales de este abandono la falta de motivación que influirá en una decisión tan importante como la de abandonar o continuar con los estudios universitarios (Repetto, E., 2008, web). Se hace necesario un aprendizaje cooperativo con experiencias que conllevan un aumento en la motivación hacia el aprendizaje, produciendo actitudes más positivas hacia el hecho de aprender, hacia sus compañeros y hacia sus profesores (Serrano, 1996: 217-244).

\subsection{La inteligencia emocional en el contexto empresarial}

José Ángel Moreno Izquierdo (2001), mostraba el resultado arrojado por varias encuestas realizadas entre el personal de selección de empresas estadounidenses, indicando que más de la mitad de las personas que trabajaban para ellos carecían de motivación para continuar aprendiendo y mejorando en su desempeño. Cuatro de cada diez trabajadores eran incapaces de operar en colaboración con sus compañeros, y sólo un 19\% de quienes aspiraban a ingresar en los puestos de rango superior tenían suficiente autodisciplina en sus hábitos laborales. 
En diversos estudios realizados en EE.UU. sobre lo que buscan los empleadores en los universitarios, las aptitudes técnicas específicas eran menos importantes que la capacidad de aprender en el puesto. Saber escuchar y comunicarse oralmente, trabajo en equipo, adaptabilidad y respuestas creativas ante los obstáculos y reveses, dominio personal, confianza en uno mismo, creatividad... y sobre todo la motivación para trabajar en pro de un objetivo, son algunas de las aptitudes y actitudes que se valoran cada vez más por parte de las empresas y los reclutadores de personal.

En cuanto a esta última capacidad de motivación cuando es visible, convierte a estas personas en excelentes colaboradores que comunican y transmiten su pasión por el trabajo, contagiando a los demás y moviéndoles en busca de soluciones.

Los líderes emocionalmente inteligentes despiertan de manera natural el interés y entusiasmo en todo el grupo, alentando una sensación de bienestar que les lleva a compartir las ideas, aprender el uno del otro, y asumir decisiones grupales. Raúl Amador León (2002) define al líder como aquel sujeto que posee un conjunto de cualidades que le permiten lograr una posición de dominio en cualquier situación, pasando el nuevo líder de la búsqueda de equilibrio, al cambio y a un intento de promover la mejora de la organización en todos los frentes.

La clave para que los universitarios puedan desarrollarse de manera eficaz en habilidades de liderazgo, consistiría en la consolidación de las competencias de la inteligencia emocional que deben estar en concordancia con sus características y estilo personal, pudiéndose aprender en el entorno de las universidades por medio de un proceso que exige un compromiso serio y constancia en el logro de este objetivo.

No obstante y a pesar de que la inteligencia emocional se convierte en un importante valor a tener en cuenta por parte de los empleadores, debe y tiene que haber un compendio entre lo racional y lo emocional, ya que como bien dicen David Caruso y Peter Salovey "el pensamiento racional necesita de las emociones y que ambos elementos no puedan separarse fácilmente" (Caruso y Salovey, 2005: 248). Es decir, debe existir un equilibrio entre las aptitudes emocionales y las destrezas académicas de los estudiantes.

\section{Metodología y desarrollo}

En lo que compete propiamente a la metodología utilizada en este trabajo y con la finalidad de recabar la mayor información posible que ayudará a apoyar la batería de conclusiones finales, se utilizaron básicamente dos métodos para recabar información.

Por una parte, y en cuanto a las fuentes de información secundarias se utilizaron fuentes bibliográfica y fuentes electrónicas de decenas de autores que tratan principalmente temas sobre la inteligencia emocional y e EEES.

En cuanto a las fuentes de información primarias y en concreto aquellas relacionadas con técnicas de índole cuantitativo, se definió un cuestionario el cual se distribuyó (usando una herramienta de encuestas que permitía su distribución de modo electrónico y fiable) tanto a estudiantes de último curso que seguían el plan Bolonia, como a profesores de universidades Madrileñas de distintas ramas de estudio. El objeto consistía en conocer su opinión sobre las mejoras generadas en Espacio Europeo de Educación Superior (EEES), y la posible incorporación de nuevas materias y dis- 
ciplinas relacionadas con la formación en competencias emocionales al modelo de competencias propuesto por el proceso de Bolonia, entendidas éstas como aquellos conocimientos y habilidades, actitudes y valores que adquieren los alumnos y que les capacitan para la resolución de problemas tanto en el contexto académico como en el profesional y personal.

Se formularon 8 preguntas, obteniéndose 65 respuestas de alumnos y 24 de profesores con los resultados que se reflejan en el siguiente gráfico.

Gráfico 1. Encuesta realizada a profesores y alumnos
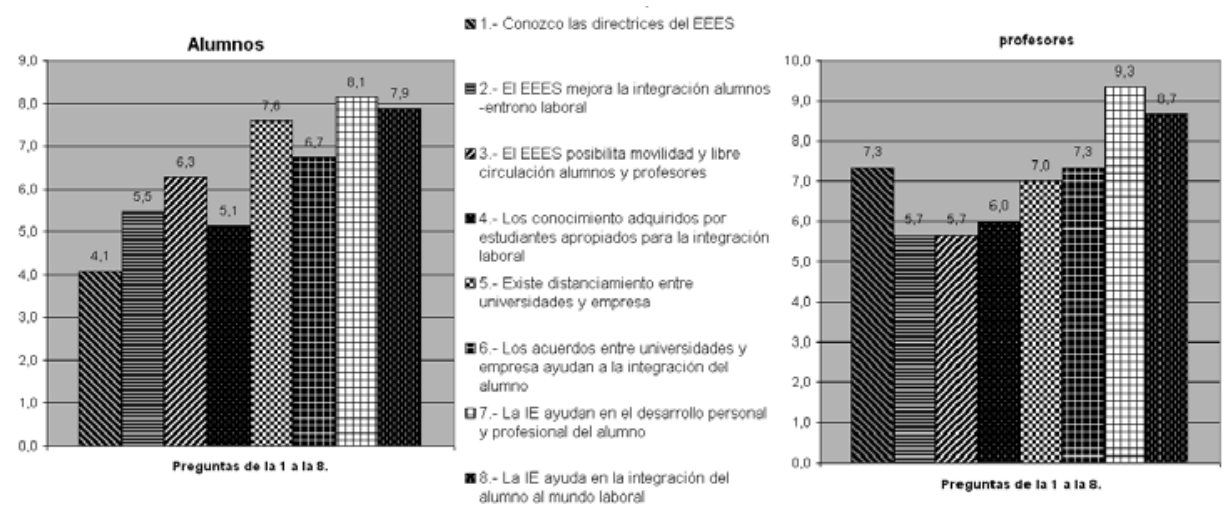

Fuente: Propia (2012)

A tenor de los resultados se observa que existe un conocimiento medio/bajo por parte de los alumnos sobre las características y directrices básicas del EEES, siendo superior este conocimiento en los profesores, aunque sin una diferencia considerable. Este hecho podría ser debido a que no se han desarrollado planes de comunicación efectivos dirigidos tanto a estudiantes como a profesores que expliquen las características básicas y ventajas del EEES.

Ambos colectivos opinan que existe un distanciamiento elevado entre las demandas de las empresas y la oferta universitaria.

Por último se produce una valoración muy positiva tanto por parte de alumnos como de profesores en cuanto a la importancia que tiene en los planes de carrera la implementación de asignaturas y materias relacionadas con la IE para la integración y el desarrollo personal y profesional de los alumnos.

\section{Conclusiones}

Aunque las universidades sepan que un cambio progresivo en sus sistemas educativos es de vital importancia para la integración efectiva de sus estudiantes al mercado laboral, pocas lo han puesto verdaderamente en práctica.

El EEES se convierte en un reto muy positivo tanto para la comunidad universitaria y empresarial, que puede constituirse como un auténtico cambio y reforma en el modo en el que se han hecho las cosas. 
Este proceso de transformación hacia un EEES ha de llevarse a cabo con la máxima colaboración y participación de las propias instituciones de enseñanza superior, así como con el máximo respeto a la diversidad de culturas y a la autonomía universitaria.

La universidad, que siempre ha jugado un papel decisivo en el desarrollo cultural, económico y social de los estados europeos, debe desempeñar una función decisiva en este nuevo escenario, liderándolo y monitorizándolo para garantizar su correcta implementación.

Se hace necesaria una nueva concepción de la formación académica, centrada en el aprendizaje del alumno, y una revalorización de la función docente del profesor universitario que incentive su motivación y que reconozca los esfuerzos encaminados a mejorar la calidad y la innovación educativa.

Las universidades deben potenciar el enfoque educativo por competencias donde se conjuguen las competencias específicas propias de la rama de estudios seleccionada por los alumnos, con competencias genéricas compartidas por todas las materias o ámbitos de conocimiento, en las cuales se podrían incluir materias que ayuden a desarrollar la inteligencia emocional de los alumnos.

Este enfoque por competencias necesita de una cooperación estrecha entre universidades y empresas, generándose acuerdos entre ambas partes, de modo que la universidad dentro de una filosofía de aprendizaje permanente se adapte a las necesidades reales de las empresas.

Poco a poco las universidades se van percatando del hecho de que el problema de la falta de rendimiento de los universitarios parece no estar tanto en el estudiante y en sus aptitudes académicas, en sus limitaciones o en sus problemas personales, y sí parece estar mucho más en manos de las propias universidades.

Las aptitudes académicas de los estudiantes van perdiendo importancia cuando las comparamos con las aptitudes o competencias socio emocionales cada vez más demandadas por los departamentos de RRHH de las empresas, ya que el coeficiente intelectual o CI (que mide las habilidades cognitivas de una persona), está pasando a ocupar un segundo plano.

Existe una opinión generalizada sobre la correcta incorporación en los planes de estudio de materias que ayuden a los universitarios a desarrollar sus competencias emocionales, convirtiéndose en un elemento indispensable para la diferenciación cualitativa de los estudiantes en un entorno laboral competitivo, pudiendo ayudarles a tener una mayor empleabilidad.

Estas materias no se deben circunscribir únicamente al entorno universitario, sino que deberían ser incorporadas en etapas educativas previas a la universidad.

\section{Referencias bibliográficas}

AMADOR LEÓN, Raúl (2002): "El líder y la cultura organizacional: su incidencia en la eficacia de la empresa". Las Palmas de Gran Canaria, Servicio de Publicaciones y Producción Documental de la Universidad de Las Palmas de Gran Canaria.

CARUSO, David y SALOVEY, Peter. (2005): El Directivo Emocionalmente Inteligente. Madrid, Editorial Algaba. 
COMISIÓN EUROPEA (2003): "El papel de las universidades en la Europa del conocimiento" en: http://www.eiconsortium.org/reports/business_case_for_ei.html. [fecha de consulta: 5 de mayo del 2010].

CONSORTIUM FOR RESEARCH ON EMOTIONAL INTELLIGENCE IN ORGANIZATIONS (2009): "The Business Case for Emotional Intelligence". en: http://www.eiconsortium.org/reports/business_case_for_ei.html. [fecha de consulta: 5 de mayo del 2010].

GARAGORRI, Xabier (2007): “Currículo basado en competencias. Aproximación en estado de la cuestión". Madrid, Aula de innovación educativa Nº161.

GARDNER, Howard (2010): Las inteligencias múltiples en el s. XXI. Barcelona, Paidós.

GOLEMAN, Daniel (2000): La práctica de la inteligencia. Barcelona, Cairos.

GOLEMAN, Daniel (2007): Inteligencia Emocional. México D.F., Ediciones B. México.

GONZALEZ FERRERAS, Julia (2006): “Tunning educational estructures in Europe II. La contribución de las universidades al proceso de Bolonia". Bilbao, Universidad de Deusto.

MORENO IZQUIERDO, José Ángel (2001): “Cuadernos de la fundación, No 65, Ética empresarial y globalización”. Madrid, Fundación Mapfre Estudios.

PEDRÓ, Francesc. (2005): "Manual d’introducción a la docéncia. Horitzó Bolonyá". Barcelona, Universidad Pompeu Fabra.

REPETTO TALAVERA, Elvira (2008): "Ponencia UNED sobre competencias socioemocionales y coeficiente intelectual" en: http://www.aprendemas.com/Noticias/html/N1612_F19072006.html: [fecha de consulta: 10 de marzo de 2011].

SALOVEY, Peter. y MAYER, John (1990): "Emotional Intelligence: Imagination, Cognition, and Personality", en: http://www.unh.edu/emotional_. [fecha de consulta: 11 de abril de 2011].

SANGRÁ, Albert. et al. (2008): "Os materiais de aprendizaxe en contextos educativos virtuais. Pautas para o deseño tecnopedagóxico". Vigo, Vicerrectoría de Formación e Innovación Educativa - Universidad de Vigo.

SERRANO GONZÁLEZ-TEJERO, José Manuel (1996): El aprendizaje cooperativo. Psicología de la instrucción I. Variables y procesos básicos. Madrid, Síntesis.

TORRE, De la., Saturnino (1993): "La creatividad en la aplicación del método didáctico". Madrid, Sevillano, M.L.

TORRE, De la., Saturnino (2009): "Estrategias creativas en el aula universitaria. Revista Digital Universitaria, ${ }^{\circ}$ 12" en http://www.revista.unam.mx/vol.10/num12 /art89/int89.htm. [fecha de consulta: 11 de abril de 2012]. 


\section{Carlos DEL VAL MERINO}

Dirección de Comunicación y Patrocinio del Instituto Cervantes cval_exmba2002@yahoo.es

Doctor por la Universidad de Málaga 